\title{
Structural evolution of a catalyst ink for fuel cells during the drying process investigated by CV-SANS
}

\author{
Takumi Kusano ${ }^{1}$, Takashi Hiroi ${ }^{1}$, Kazuki Amemiya ${ }^{2}$, Masaki Ando ${ }^{2}$, Tsuyoshi Takahashi ${ }^{2}$ \\ and Mitsuhiro Shibayama ${ }^{1}$
}

Polymer electrode fuel cells are fabricated by drying a 'catalyst ink', that is, a mixture of carbon, ionomers, Pt and water, and it is of particular importance to elucidate the structural evolution of the catalyst ink during its drying process. The structural evolution of catalyst ink was investigated by contrast-variation small-angle neutron scattering. The scattering functions of the catalyst ink at various concentrations were successfully decomposed to the corresponding partial structure factors $S_{\mathrm{CC}}(q), S_{\mathrm{PP}}(q)$ and $S_{\mathrm{CP}}(q)$ for all ink concentrations, where the subscripts $\mathrm{C}$ and $\mathrm{P}$ denote the carbon and polymer, respectively, and $q$ is the magnitude of the scattering vector. The cross term $S_{C P}(q)$ indicates that the microscopic structure of the catalyst ink retains the carbon agglomerates surrounded by an ionomer shell during the drying process. $S_{\mathrm{cc}}(q)$ and $S_{\mathrm{PP}}(q)$ indicate that a catalyst ink is formed with carbon agglomerates of core radius of $\sim 42 \mathrm{~nm}$ and an ionomer shell of thickness $>8 \mathrm{~nm}$. Both the size of the carbon agglomerates and the thickness of the ionomer shells decrease with increasing ink concentration, which is attributed to the exclusion of solvent molecules from the carbon and ionomer agglomerates during the drying process.

Polymer Journal (2015) 47, 546-555; doi:10.1038/pj.2015.36; published online 17 June 2015

\section{INTRODUCTION}

Polymer electrode fuel cells (PEFCs) are one of the most promising energy sources. ${ }^{1}$ Because PEFCs are zero-emission devices that convert chemical energy into electricity through a chemical reaction between hydrogen and oxygen, PEFCs are the ultimate clean energy technology. The energy efficiency is more than $30 \%$, which is much higher than that of internal combustion engines. Hence, the development of PEFCs has been accelerated in the last few decades in the industrial context, particularly for fuel cell vehicles. However, several problems remain to be solved, such as the reduction of the cost of the Pt catalyst and the durability of the cell. To solve these problems, the fabrication process of the electrodes of the PEFC has to be optimized. The electrodes of PEFCs are often manufactured from 'catalyst inks' consisting of carbon, platinum, water and ionomers..$^{2-4}$ A catalyst ink is prepared by mixing ionomer solutions, carbon powder with the Pt catalyst, and a dispersing solvent, such as water and alcohol. The electrodes are manufactured by coating the catalyst ink onto a substrate. Then, the electrodes are transferred to a proton exchange membrane, such as a Nafion membrane, and form a membrane-andelectrode assembly. Because this manufacturing process includes various steps, the performance of PEFCs greatly depends on their structure and on the preparation recipe of the electrodes.

Because understanding the structure of PEFCs is one of the key issues to improve the efficiency of the cell, a large number of studies have been carried out, ${ }^{5-8}$ especially relative to the structure of the ionomers. Aldebert et al. ${ }^{9}$ reported that the ionomers formed rod-like aggregates in an aqueous or alcohol solution. Furthermore, Gebel et al. ${ }^{10-12}$ elucidated the structural transition of ionomers from rod-like aggregates to percolated ionic channels by a drying process. However, several studies have been carried out on the structure of catalyst inks or electrodes. Recently, an extensive review on fuel cell catalyst layers was reported by Holdcroft. ${ }^{4}$ According to the results of molecular dynamics simulations or transmission electron microscopy, the electrode consists of 'agglomerates, ${ }^{13,14}$ which are composed of several primary carbon particles and 'primary pores' of $2-20 \mathrm{~nm}$ diameter covered by an ionomer shell a few nanometers long. These agglomerates form a higher structure called an 'aggregate' by assembling together. The ionomer shells are expected to exhibit hydrophilic surfaces formed by the hydrophilic groups of the ionomer, which assist the solvation of carbon agglomerates in an aqueous solution. ${ }^{15}$ Moreover, it has been suggested that there is an inhomogeneous distribution of the ionomers in the electrodes (catalyst layers). ${ }^{16}$ This inhomogeneity in the electrode structure is important to control the balance of proton and gas transportation.

In our previous study, we used contrast-variation small-angle neutron scattering (CV-SANS) to investigate the structure of PEFCs. ${ }^{17}$ CV-SANS is a powerful technique for investigating the structure of multi-component materials because it allows decomposition of the scattering intensity functions into partial scattering functions, $S_{i j}(q){ }^{18,19}$ Here, $i$ and $j$ refer to the components, such as carbon $(\mathrm{C})$, polymer $(\mathrm{P})$ and water $(\mathrm{W})$, and $q$ is the magnitude of the scattering vector. The following facts were uncovered after precise determination of the scattering length density (SLD) of each component, followed by SANS experiments at various solvent 
compositions of different SLDs: (1) The microscopic structure of the catalyst ink consists of dendric clusters of carbon agglomerates surrounded by ionomers. (2) The SANS function of ionomer solutions has an ionic cluster peak around $q \approx 0.05 \AA^{-1}$, which maintains its structure even in a mixture of ink and carbon/Pt. (3) The cross term $S_{\mathrm{CP}}(q)$ indicates a percolated structure of carbon clusters mediated by ionomers. The presence of this ionic-cluster path seems to have a key role in the performance of the solid polymer electrodes in polymer electrolyte fuel cells.

However, because the concentration of the catalyst ink investigated in the previous paper was of the order of $1 \mathrm{wt} \%$ polymer, it was not clear how the electrode structure was evolving during the drying process. Hence, it was desired to understand how a solid electrode is formed and how one can improve the performance of the PEFCs. Neither electron microscopy, nor ultraviolet-visible spectroscopic methods can be used for monitoring the change in the structure and concentration of the components during the drying process because of the wet nature of the sample and a strong light absorption, respectively. The information obtained from small-angle X-ray scattering is limited in the study of multi-component systems. Hence, we again used the CV-SANS method to study the structural evolution of a catalyst ink during the drying process. Although we encountered various difficulties in conducting the CV-SANS experiments on the catalyst ink, such as the precise determination of the sample thickness, we successfully obtained interesting information, which may benefit the further understanding of the structure of PEFCs.

\section{Theoretical}

$C V$-SANS method. In the case of three-component systems, the scattering intensity function is written as follows, based on the assumption of incompressibility:

$$
\begin{aligned}
I(q)= & \left(\rho_{\mathrm{A}}-\rho_{\mathrm{S}}\right)^{2} S_{\mathrm{AA}}(q)+\left(\rho_{\mathrm{B}}-\rho_{\mathrm{S}}\right)^{2} S_{\mathrm{BB}}(q) \\
& +2\left(\rho_{\mathrm{A}}-\rho_{\mathrm{S}}\right)\left(\rho_{\mathrm{B}}-\rho_{\mathrm{S}}\right) S_{\mathrm{AB}}(q)
\end{aligned}
$$

Here, $\rho_{i}\left(=\sum_{j} b_{j} / v_{j}\right)$ indicates the scattering length density of each component $i(i=\mathrm{A}, \mathrm{B}, \mathrm{S}), b_{j}$ is the scattering length of the $j$-th atom and $v_{i}$ is the molecular (segment) volume of component $i$. Here, A, B and $\mathrm{S}$ denote component $\mathrm{A}$, component $\mathrm{B}$ and the solvent (or the medium), respectively. $S_{i l}(q)$ denotes a partial scattering function. $S_{\mathrm{AA}}$ $(q)$ and $S_{\mathrm{BB}}(q)$ are called the self-terms, representing the selfcorrelation of component $\mathrm{A}$ and $\mathrm{B}$, respectively. $S_{\mathrm{AB}}(q)$ is called the cross-term, representing the cross-correlation between components $\mathrm{A}$ and B. By conducting a series of SANS experiments with solvents having different scattering-length densities $\rho_{\mathrm{s}}$, one obtains a series of scattering functions, $I_{1}(q), I_{2}(q), I_{3}(q), \ldots, I_{n}(q)$. By solving these equations with the singular value decomposition method, a

core-shell
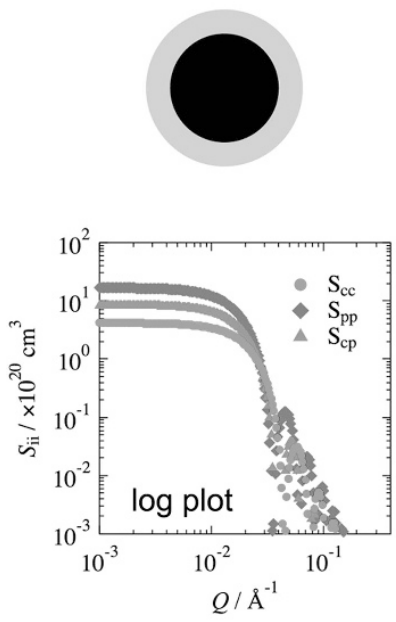

b
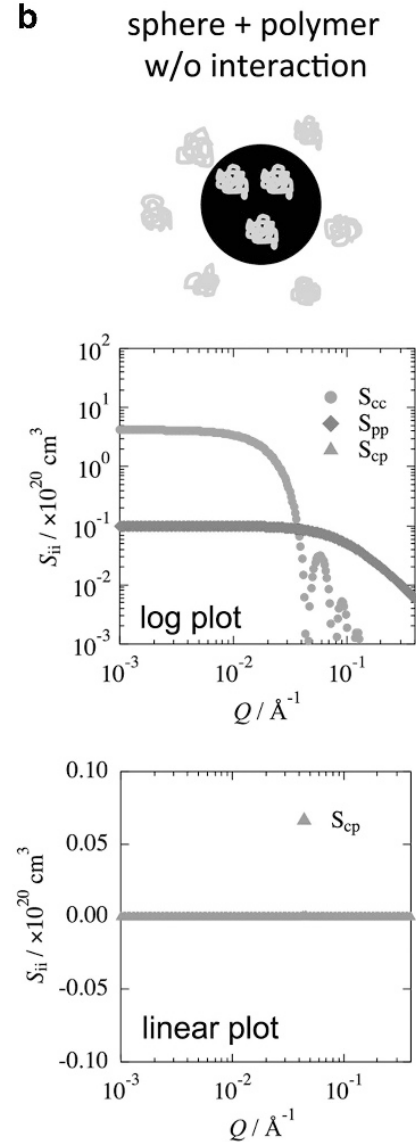

C
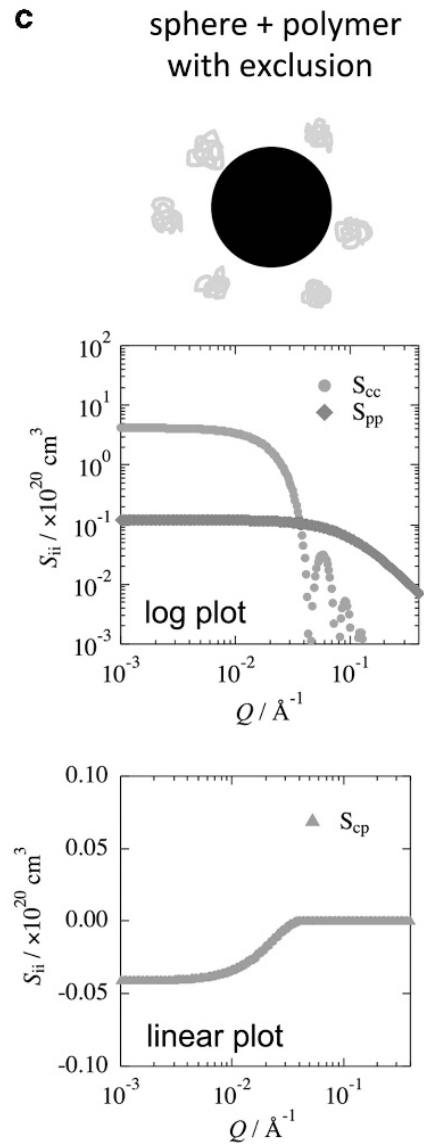

Figure 1 Schematic illustrations showing three models: (a) core-shell model, a mixture of spheres and polymers with no interactions and (b) with excluded volume interactions, and (c) corresponding partial structure factors, $S_{i j}(q)$ s. The meaning of $S_{A B}(q)$ is clearly shown in the lower row. We used the following parameters for the corresponding models: (a) core radius $=100 \AA$, shell thickness $=26 \AA$, concentrations of the core and shell $=1$ vol\% each, (b) and (c) radius of sphere $=100 \AA$, polymer $R_{\mathrm{g}}=10 \AA$, concentrations of the sphere and polymer $=1$ vol\% each. A full color version of this figure is available at Polymer Journal online. 
one obtains $S_{i l}(q)$. This is called the CV-SANS method. The singular value decomposition method is the factorization of a $3 \times n$ matrix. This method is analogous to solving a system of three simultaneous equations, for which the number of equations and solutions is the same. However, singular value decomposition requires a larger number of equations $(n)$ to obtain the three solutions because of uncertainty (or errors) in the equations. The details of CV-SANS are described elsewhere. ${ }^{18-20} \mathrm{CV}$-SANS is advantageous in that it allows one to evaluate the cross term, that is, $S_{\mathrm{AB}}(q)$. Because it provides information about the relationship between phases $\mathrm{A}$ and $\mathrm{B}$, such as the interface, CV-SANS is often used in analyses of multi-component systems. Figure 1 schematically shows the models (top row) of (a) the core $(\mathrm{C})$-shell $(\mathrm{P})$ and a mixture of spheres $(\mathrm{C})$ and polymers $(\mathrm{P})$ with no interaction and (b) of the same system with an excluded volume interaction and (c) the corresponding $S_{\mathrm{ij}}(q)$ values (the middle and lower rows). Here, the most important observation is the shape and the sign of $S_{\mathrm{CP}}(q)$. If polymer chains are adsorbed on the surface of the cores, $S_{\mathrm{CP}}(q)$ is positive (the core-shell model; model a). However, if there is no interaction between $\mathrm{C}$ and $\mathrm{P}$, the resultant $S_{\mathrm{CP}}(q)$ is zero irrespective of $q$ (model b). If there is excluded volume interaction, $S_{\mathrm{CP}}(q)$ is negative in the low $q$ region due to the hard core potential of component $\mathrm{C}$ (model c). In the latter part of this study, we will discuss the structure of the catalyst ink with $S_{\mathrm{CP}}(q)$.

Sample thickness evaluation via transmission. Sample thickness measurements in scattering experiments are usually easy to perform and can be directly acquired with a mechanical, electrical or optical micrometer. However, if a sample is contained in a sealed cell, the sample thickness cannot be measured. Here, we propose a method to indirectly estimate the sample thickness by using neutron transmission measurements. Hence, the sample thickness $t(\mathrm{~cm})$ was estimated by measuring the neutron transmission $T$ and calculating the total crosssection of the samples, $\sum_{\text {tot }}\left(\mathrm{cm}^{-1}\right)$. According to the Beer-Lambert Law, the sample thickness $t$ is given by:

$$
\begin{aligned}
& t=-\frac{1}{\sum_{\text {tot }}} \ln T \\
& \sum_{\text {tot }} \text { is given by: } \\
& \sum_{\text {tot }}=\sum_{i} \rho_{i} \sigma_{\text {tot }, i}
\end{aligned}
$$

Here, $\rho_{i}\left(\mathrm{~cm}^{-3}\right)$ and $\sigma_{\text {tot, } i}\left(\mathrm{~cm}^{2}\right)$ are the number density and total cross-section of the scattering element $i$. For compound $i, \rho_{i}$ is given by:

$$
\rho_{i}=N_{\mathrm{A}} \frac{d_{i}}{m_{i}}
$$

where $d_{i}\left(\mathrm{~g} \mathrm{~cm}^{-3}\right)$ and $m_{i}\left(\mathrm{~g} \mathrm{~mol}^{-1}\right)$ are the mass density and molecular weight of compound $i$, and $N_{\mathrm{A}}$ is Avogadro's number. The total cross-section is the sum of the coherent scattering cross sections $\sigma_{\mathrm{coh}, i}$, the incoherent scattering cross-section $\sigma_{\mathrm{inc}, i}$, and the absorption cross-section $\sigma_{\mathrm{abs}, i}$. Hence, $\sigma_{\mathrm{tot}, i}$ is given by:

$$
\sigma_{\mathrm{tot}, i}=\sigma_{\mathrm{coh}, i}+\sigma_{\mathrm{inc}, i}+\sigma_{\mathrm{abs}, i}
$$

Therefore, the sample thickness can be calculated from the sample transmission and total cross-section if the sample composition is known. The validity of this estimation is examined in this work.

\section{EXPERIMENTAL PROCEDURE}

\section{Samples}

A series of catalyst inks was prepared by mixing $30 \mathrm{wt} \%$ platinum-loaded carbon black (TEC10V30E-HT, TANAKA KIKINZOKU KOGYO K.K.) and a
Table 1 Sample code and volume concentration of the components of the catalyst inks used for the SANS experiments

\begin{tabular}{lccc}
\hline Code & $\phi_{C}$ & $\phi_{P}$ & $\phi_{P t}$ \\
\hline Stage 1 & 0.013 & 0.015 & 0.0006 \\
Stage 2 & 0.027 & 0.031 & 0.0012 \\
Stage 3 & 0.040 & 0.046 & 0.0018 \\
Stage 4 & 0.053 & 0.061 & 0.0024 \\
\hline
\end{tabular}

Nafion (DE2020, Du Pont, 21\% aqueous solution) or IonomerA dispersion (IonomerA is a PFSI-type of ionomer having similar properties to that of Nafion but with improved proton conductivity). The primary particle size and the specific surface of the carbon particles were $30 \mathrm{~nm}$ and $117 \mathrm{~m}^{2} \mathrm{~g}^{-1}$, respectively. The volume concentrations of the stock solution of the catalyst ink were $\phi_{\mathrm{C}}=0.013$ (for carbon), $\phi_{\mathrm{P}}=0.015$ (for the ionomer) and $\phi_{\mathrm{Pt}}=0.0006$ (for Pt). As described in Table 1, four samples having different concentrations, with ratios of 2 (stage 2), 3 (stage 3 ) and 4 (stage 4 ), were prepared by partial drying and were sealed in custom-made SANS quartz cells. For the CV-SANS experiments, five sets of samples with different $\mathrm{H}_{2} \mathrm{O} / \mathrm{D}_{2} \mathrm{O}$ ratios, that is, $\phi_{\mathrm{D} 2 \mathrm{O}}=0.55,0.65,0.75,0.80$ and 0.85 , were prepared together in a temperature-and-humidity-controlled large chamber to maintain the same structure in the same set of samples. The sample codes and concentrations are listed in Table 1. SANS studies for catalyst ink concentrations higher than Stage 4 could not be carried out because of crack formation.

\section{Small-angle neutron scattering}

The SANS experiments were performed on a High-flux Advanced Neutron Application Reactor at the Korea Atomic Energy Research Institute, Daejeon, South Korea. The samples were irradiated with a monochromatic cold neutron beam with an average neutron wavelength of $6.00 \AA$, and the scattered neutrons were counted with a two-dimensional detector. Two sample-to-detector distances, 2 and $17.5 \mathrm{~m}$, were used to cover a $q$ range between 0.003 and $0.4 \AA^{-1}$, where the magnitude of the scattering vector $q$ is defined by $q=4 \pi \sin \theta / \lambda$ ( $\lambda$ and $2 \theta$ represent the wavelength and the scattering angle, respectively). All measurements were performed at room temperature. After appropriate corrections for open-beam scattering, transmission, sample thickness, detector inhomogeneities and incoherent scattering, the corrected scattering intensity functions were normalized to the absolute intensity scale by using the direct-beam method.

\section{Sample thickness determination}

In this study, the sample thicknesses for SANS, $t$, could not be measured directly because the SANS samples were prepared by pre-drying in the SANS cells followed by sealing. The sample thicknesses of the catalyst ink at $\phi_{\mathrm{D} 2 \mathrm{O}}=0.85,0.8,0.75,0.65$ and 0.55 were estimated to be $t_{\text {calc }}=0.39,0.39$, $0.38,0.37$ and $0.38 \mathrm{~cm}$, respectively. The relative error is defined by:

$$
\text { error } \equiv \frac{t_{\text {meas }}(=0.4 \mathrm{~cm})-t_{\text {calc }}}{t_{\text {meas }}(=0.4 \mathrm{~cm})}
$$

The errors estimated from $\sum_{\text {tot }}$ and $T$ in Equations (3) and (2), respectively, lie within only $7.5 \%$ of the measured values. Hence, we successfully estimated the sample thickness of a series of catalyst ink samples with a known thickness $(0.4 \mathrm{~cm})$ from the neutron transmission measurements.

\section{RESULTS AND DISCUSSION}

\section{Contrast-variation SANS}

In a previous study, we evaluated the SLD of carbon and Nafion. ${ }^{17}$ The SLDs for carbon and Nafion were estimated to be $\rho_{\mathrm{C}}=6.18 \times 10^{10} \mathrm{~cm}^{-2}$ and $\rho_{\mathrm{P}}=3.25 \times 10^{10} \mathrm{~cm}^{-2}$, respectively. Figure 2 shows the SLD for carbon, Nafion and the solvent (a mixture of $\mathrm{D}_{2} \mathrm{O}$ and $\mathrm{H}_{2} \mathrm{O}$ ), together with the water compositions used for the 
CV-SANS experiments (open symbols with arrows). Figure 3a shows the SANS profiles of the catalyst inks made of aqueous solutions of carbon and Nafion with various $\phi_{\mathrm{D} 2 \mathrm{O}}$ for the stock solution $\left(\phi_{\mathrm{C}}=0.013\right.$ and $\phi_{\mathrm{P}}=0.015$, Stage 1$)$. For $\phi_{\mathrm{D} 2 \mathrm{O}}=0.55, I(q)$ shows a shoulder (Guinier region) in the scattering intensity in the range of $0.003<q<0.01 \AA^{-1}$. Here, the Guinier region is characterized by a

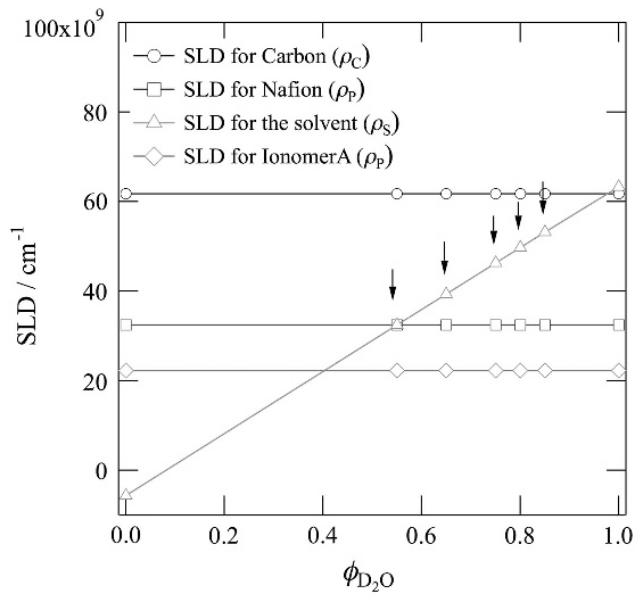

Figure 2 Variations of the scattering length density (SLD) as a function of the solvent composition, $\phi_{\mathrm{D} 2 \mathrm{O}}$. A full color version of this figure is available at Polymer Journal online. crossover in the scattering function at low $q$, which obeys different power-laws. For $\phi_{\mathrm{D} 2 \mathrm{O}}=0.55$, the SLD for the solvent $\left(\rho_{\mathrm{S}}\right)$ is $3.25 \times 10^{10} \mathrm{~cm}^{-2}$, which is nearly equal to the SLD for Nafion $\left(\rho_{\mathrm{P}}=3.25 \times 10^{10} \mathrm{~cm}^{-2}\right)$. Hence, the $I(q) \mathrm{s}$ at $\phi_{\mathrm{D} 2 \mathrm{O}}=0.55$ indicates a carbon-dominant scattering. According to a previous study, ${ }^{17}$ this intensity in the $0.003<q<0.01 \AA^{-1}$ range (Guinier region) is attributed to carbon agglomerates, that is, a small number of assemblies of primary carbon particles. The power-law behavior at high $q(0.01<q)$ will be discussed in a later section. With increasing $\phi_{\mathrm{D} 2 \mathrm{O}}$, the scattering intensity decreases, which means that carbon scattering decreases gradually due to a decrease in the SLD contrast between $\rho_{\mathrm{S}}$ and $\rho_{\mathrm{C}}$. For $\phi_{\mathrm{D} 2 \mathrm{O}}=0.85$, where Nafion scattering becomes dominant, $I(q)$ is a monotonic decreasing function of $q$. This behavior indicates that the size of the aggregates, dominantly represented by Nafion (that is, assemblies of carbon agglomerates covered with Nafion), is larger than nanometer scales. In this manner, it can be noted that the $I(q)$ of catalyst ink is a sensitive function of $\phi_{\mathrm{D} 2 \mathrm{O}}$ not only for the magnitude but also for the shape of the function.

Figures $3 \mathrm{~b}$ and $\mathrm{c}$ show the SANS profiles of the catalyst ink concentrated to (b) Stage $2\left(\phi_{\mathrm{C}}=0.027\right.$ and $\left.\phi_{\mathrm{P}}=0.031\right)$ and (c) Stage $3\left(\phi_{\mathrm{C}}=0.040\right.$ and $\left.\phi_{\mathrm{P}}=0.046\right)$. At these concentrations, the behavior of the SANS profile is almost the same as that of Stage 1. However, the SANS profile for Stage 3 at $\phi_{\mathrm{D} 2 \mathrm{O}}=0.85$ shows a Guinier region in the $0.003<q<0.01 \AA^{-1}$ range, which was not observed for Stage 1 at $\phi_{\mathrm{D} 2 \mathrm{O}}=0.85$. This behavior indicates that the agglomerates became smaller during the drying process. Figure $3 \mathrm{~d}$ shows the SANS profiles of the catalyst ink concentrated to Stage $4 \quad\left(\phi_{\mathrm{C}}=0.053\right.$ and

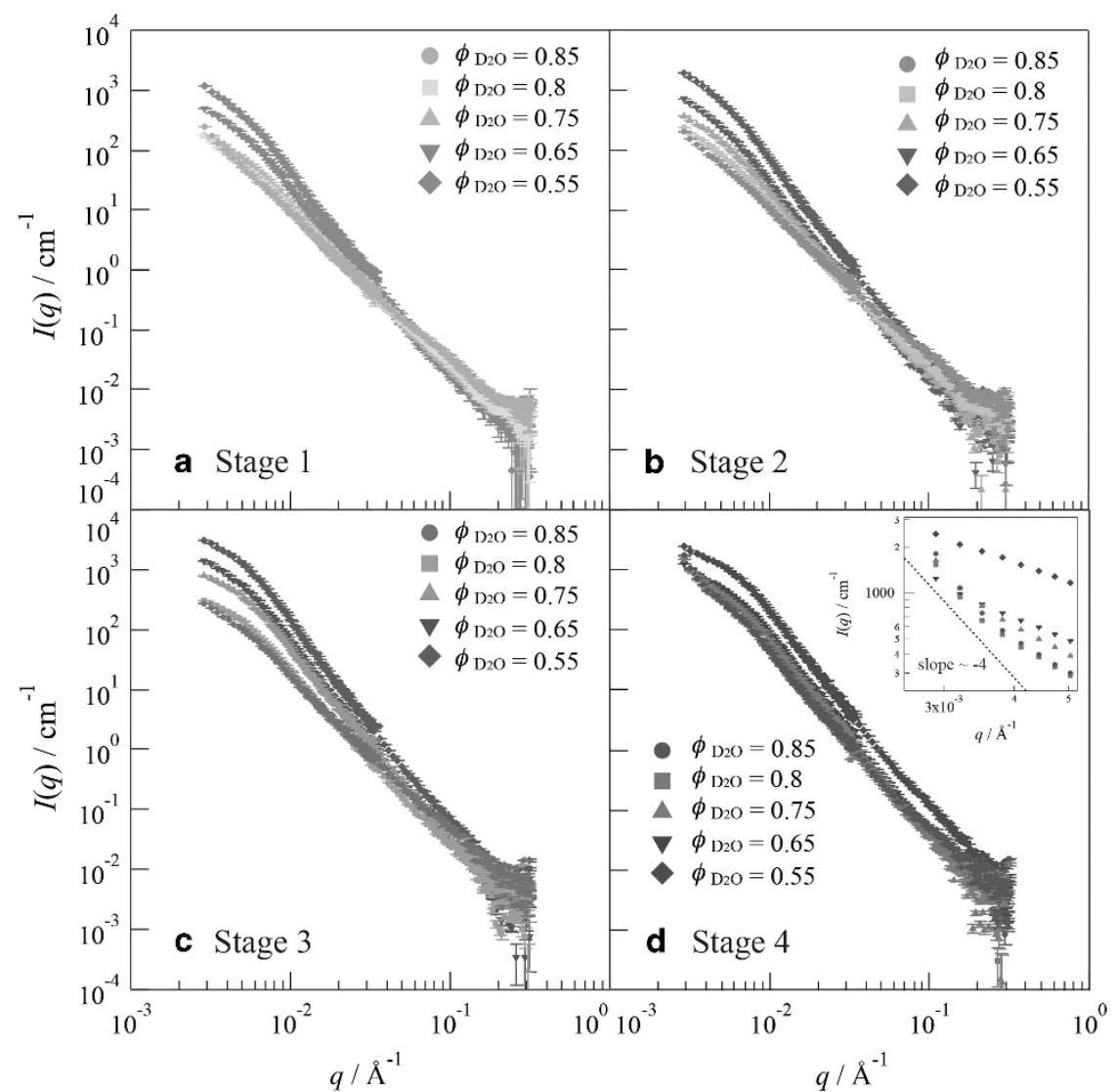

Figure 3 Series of SANS profiles for the catalyst ink with carbon and Nafion measured with different solvent compositions, $\phi_{D 20}$. The concentrations of the catalyst ink are those for (a) Stage 1, (b) Stage 2, (c) Stage 3 and (d) Stage 4. A full color version of this figure is available at Polymer Journal online. 
$\left.\phi_{\mathrm{P}}=0.061\right)$. For $\phi_{\mathrm{D} 2 \mathrm{O}}=0.55$, a Guinier region was observed at a higher $q$ region $\left(0.004<q<0.015 \AA^{-1}\right)$ than that for Stage 3, which indicates that the size of the carbon agglomerates for Stage 4 became smaller than that of the agglomerates in Stage 3. On the other hand, for all $\phi_{\mathrm{D} 2 \mathrm{O}}$, an abrupt increase in the scattering intensity in the low $q$ region $\left(0.003<q<0.004 \AA^{-1}\right.$; indicated with a dashed line) was observed. This increase showed a $I(q) \sim q^{-4}$ behavior. A possible reason for this behavior is aggregate formation, for example, aggregates consisting of Nafion-covered and carbon agglomerates, in the sample during the drying process. It is reported that the USANS profiles of a Nafion membrane also exhibit a $I(q) \sim q^{-4}$ behavior in very low $q$ region (below $q=0.001 \AA^{-1}$ ), which originates from the clustering of the crystal regions, thus forming larger scale structures. ${ }^{5}$

\section{Singular value decomposition and reconstruction of $I(q)$}

To elucidate the structure of the catalyst ink, it is necessary to decompose the observed SANS function. A singular value decomposition method was applied on the set of $I(q)$ s shown in Figure 3 to obtain the partial structure factors. According to our previous study, ${ }^{17}$ the scattering of Pt cannot be observed because the amount of $\mathrm{Pt}$ is too small to be detected by SANS. Therefore, we do not discuss the role of Pt in the agglomerates in this study, though it is reported that Pt also influences the structure of agglomerates. ${ }^{13}$ Figures $4 \mathrm{a}-\mathrm{d}$ shows a series of SANS functions: the observed (open symbols) and reconstructed (lines) SANS functions for Stages 1 to 4, respectively. Note that the reconstructed SANS functions well reproduced the observed SANS functions, assuring the validity of the decomposition.

\section{Evaluation of partial structure factors}

Figures $5 \mathrm{a}-\mathrm{c}$ show the three partial structure factors, (a) $S_{\mathrm{CC}}(q)$, (b) $S_{\mathrm{PP}}(q)$ and $(\mathrm{c}) S_{\mathrm{CP}}(q)$, for Stages 1 to 4 , respectively. We used the same SLD values as in our previous study to extract the partial structure factors. ${ }^{17} S_{\mathrm{CC}}(q), S_{\mathrm{PP}}(q)$ and $S_{\mathrm{CP}}(q)$ increased significantly during the drying process due to the increase in ink concentration. For $S_{\mathrm{CC}}(q)$ and $S_{\mathrm{PP}}(q)$, the shoulder of the partial structure factors at $0.003<q<0.01 \AA^{-1}$ shifted to a higher $q$ region $(0.004<q<0.015$ $\AA^{-1}$ ) during the drying process, indicating that the size of the carbon and Nafion agglomerates decreased with increasing ink concentrations. $S_{\mathrm{CP}}(q)$ represents the correlation between carbon and the polymer. If the agglomerates consist of a polymer shell and carbon core, $S_{\mathrm{CP}}(q)$ is given by ${ }^{19}$

$$
\begin{aligned}
& S_{\mathrm{CP}}(q) \approx n_{C}\left(\phi_{\mathrm{pl}}-\phi_{\mathrm{pn}}\right) V_{\mathrm{pl}} \Phi_{\mathrm{pl}}(q) V_{\mathrm{C}} \Phi_{\mathrm{C}}(q) \\
& -n_{\mathrm{C}} \phi_{\mathrm{pn}} V_{\mathrm{C}}^{2} \Phi_{\mathrm{C}}^{2}(q)
\end{aligned}
$$

where $\phi_{\mathrm{pl}}, \phi_{\mathrm{pn}}, n_{\mathrm{C}}, V_{\mathrm{C}}$ and $V_{\mathrm{pl}}$ are the local volume fraction of polymer at the adsorbed layer, the volume fraction of the isolated polymer, the number density of agglomerates, the volume of carbon agglomerates and the volume of carbon agglomerates with a polymer layer, respectively. $\Phi_{\mathrm{C}}$ and $\Phi_{\mathrm{pl}}$ are the scattering amplitudes of the form factor for carbon agglomerates and for carbon agglomerates with a polymer layer, respectively. In catalyst inks, the ionomer forms a polymer layer around the carbon agglomerates, as reported by Holdcroft ${ }^{4}$ and by us in the previous study. ${ }^{17}$ Therefore, $\phi_{\mathrm{pl}}$ is much larger than $\phi_{\mathrm{pn}}$. Note that in the CV-SANS, the cross term $S_{\mathrm{CP}}(q)$

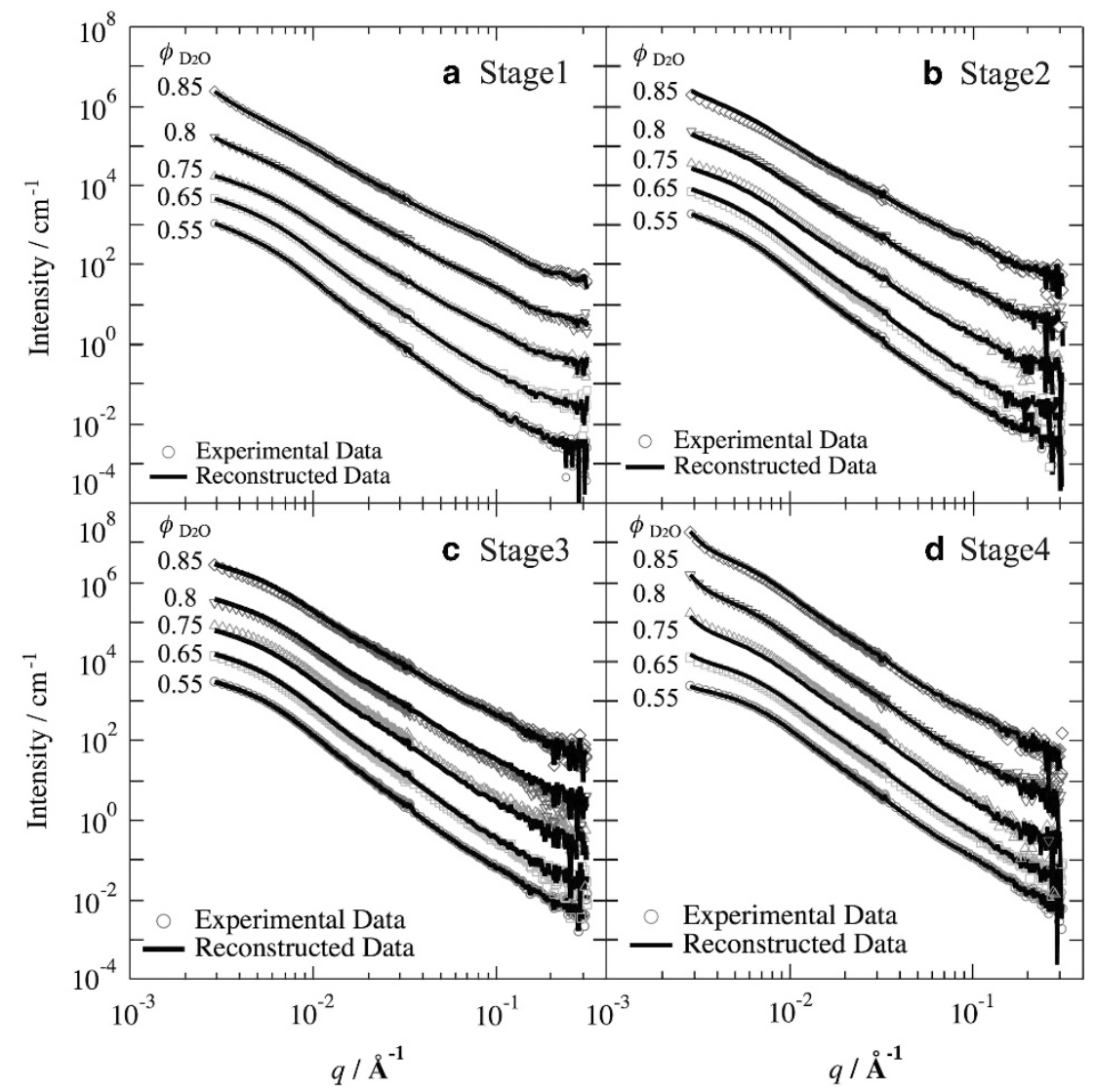

Figure 4 Observed (open symbols) and reconstructed (lines) SANS profiles for the catalyst ink with carbon and Nafion for (a) Stage 1, (b) Stage 2, (c) Stage 3 and (d) Stage 4. The profiles are vertically shifted to avoid overlapping. A full color version of this figure is available at Polymer Journal online. 
shows an important signature for components A and B, as discussed in the Theoretical section and elsewhere. ${ }^{18}$ If the thickness of the shell $\left(t_{\text {shell }}\right)$ is large enough, $S_{\mathrm{CP}}(q)$ shows a positive value. However, in the presence of a small shell or if an excluded volume interaction is present between the core and the polymer layer, $S_{\mathrm{CP}}(q)$ shows a negative value. The experimental results show that the $S_{\mathrm{CP}}(q)$ of the catalyst ink shows a positive value for all ink concentrations, which

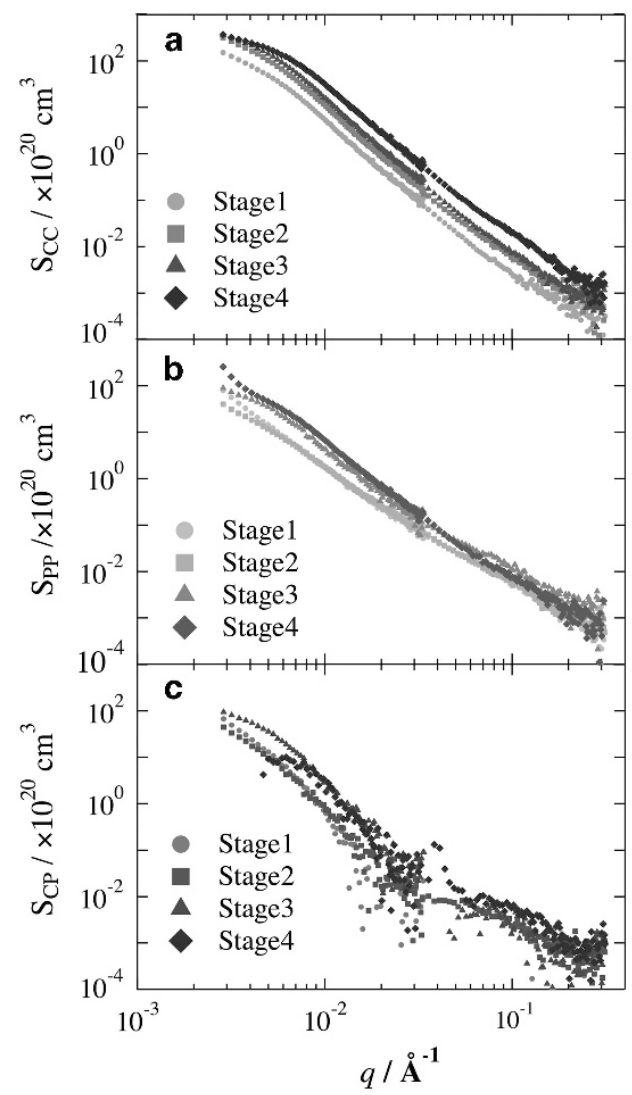

Figure 5 Decomposed partial structure factors, (a) $S_{C C}(q)$, (b) $S_{p p}(q)$ and (c) $S_{C P}(q)$ with varying ink concentration. A full color version of this figure is available at Polymer Journal online.

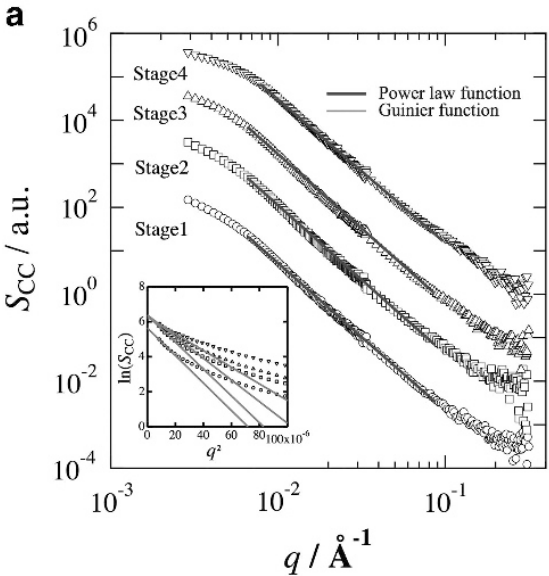

implies that the catalyst ink is composed of a core-and-shell structure, corresponding to the carbon agglomerates and a thick Nafion layer, respectively. Therefore, $S_{\mathrm{PP}}(q)$ does not represent the scattering functions of Nafion agglomerates, but rather, the scattering functions of Nafion shells.

Figures $6 \mathrm{a}$ and b show the $S_{\mathrm{CC}}(q)$ and $S_{\mathrm{PP}}(q)$, that is, the carbon scattering and Nafion scattering, respectively, of the sample. Note that each SANS function was shifted vertically to avoid overlap. Although a fitting with Equation (7) by assuming a spherical shell structure was tried for $S_{\mathrm{PP}}(q)$, it was not successful. Because $S_{\mathrm{PP}}(q)$ resembles $S_{\mathrm{CC}}(q)$, a Guinier fit analysis was conducted for both $S_{\mathrm{CC}}(q)$ and $S_{\mathrm{PP}}(q)$. The red solid lines of the insets are a fit with a Guinier function in the low $q$ region and the blue solid lines are a fit with a power law function $\left(0.008<q<0.1 \AA^{-1}\right)$. Note that the radius of gyration of thin spherical shells of radius $R$ is $R_{\mathrm{g}}=R$ and that of spheres is $R_{\mathrm{g}}=(3 / 5)^{1 / 2} R$. Hence, if the $R_{\mathrm{g}}$, Nafion obtained from $S_{\mathrm{PP}}(q)$ represents the radius of gyration of a spherical shell, $R_{\mathrm{g}}$ should be $\sim 30 \%\left(\approx(5 / 3)^{1 / 2}\right)$ larger than that of spheres. This relationship is not observed in this work because $R_{\mathrm{g}, \text { Nafion }} \approx R_{\mathrm{g}, \mathrm{Carobn}} \approx 40 \mathrm{~nm}$. This means that the Nafion chains permeated the carbon agglomerates via nanopores and formed Nafion agglomerates. We obtained the radius of gyration $R_{\mathrm{g}}$ and the surface fractal $D_{\mathrm{S}}$ from $S_{\mathrm{CC}}(q)$ and $S_{\mathrm{PP}}(q)$. Furthermore $S_{\mathrm{PP}}(q)$ showed an additional scattering as an inflection point at a high $q$ region $\left(0.03<q<0.2 \AA^{-1}\right.$; indicated by an arrow) due to ionic clusters of the ionomer (Nafion) in the matrix. The green solid line in Figure $6 \mathrm{~b}$ shows the scattering profile of a Nafion solution. ${ }^{17}$ The scattering profile of the catalyst ink showed an inflection point at the position where the Nafion solution exhibited a peak. A similar peak is commonly observed in ionomer solutions ${ }^{21}$ and PEFCs. ${ }^{6,17}$ This peak corresponds to the inter-ionic cluster distance, $d_{\mathrm{P}} \approx 17 \mathrm{~nm}$ (for Stage 1). Therefore, $d_{\mathrm{P}}$ can be estimated from the $q$-position of the inflection point.

The estimated $R_{\mathrm{g}}$ values from the Guinier fit are shown in Figure 7a. The size of the carbon agglomerates decreased upon drying. Because the sign of $S_{\mathrm{CP}}(q)$ is positive, it is deduced that the solute is composed of carbon/Nafion agglomerates and a Nafion shell. We evaluated the thickness of the Nafion layer, $t_{\text {shell }}$, by subtracting $R_{\mathrm{g}}$, carbon from $R_{\mathrm{g}, \text { Nafion. }}$. The estimated $t_{\text {shell }}$ is also shown in Figure 7a. As shown in the figure, the thickness of the Nafion shell also decreases upon drying. According to a previous study, ${ }^{14}$ the ionomer layers are a few nanometers thick in the catalyst layer (electrode). The results are

b

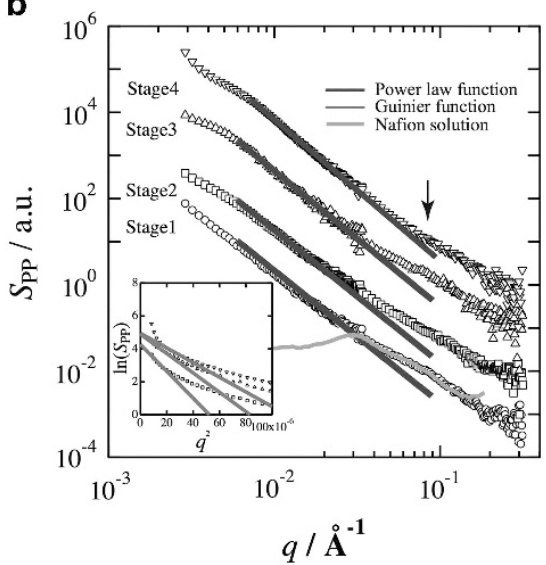

Figure 6 Results of the curve fitting of (a) $S_{C C}(q)$ and (b) $S_{p p}(q)$ with a Guinier function (dashed lines) and a power law function (solid lines). The profiles are vertically shifted to avoid overlapping. A full color version of this figure is available at Polymer Journal online. 
consistent with the values discussed by Holdcroft and in the reference therein. ${ }^{4}$

Next, let us discuss the surface of the agglomerates, based on the asymptotic behavior of the SANS data. As is well known, the SANS functions is related to the fractal dimension of a surface fractal, $D_{S}$, by a power law, as given by: ${ }^{22}$

$$
I(q \rightarrow \infty) \propto q^{D_{\mathrm{s}}-6}
$$

The fractal dimension for a surface fractal, $D_{S}$, should be in the range of 2 (smooth surface) or 3 (rough surface). For the catalyst ink system, the power law of $S_{\mathrm{CC}}(q)$ and $S_{\mathrm{PP}}(q)$ at high $q(0.008<q<0.1$ $\AA^{-1}$ ) reflects the surface dimension of carbon and ionomer (Nafion) agglomerates, respectively. The estimated $D_{\mathrm{S}}$ from a power law fitting is shown in Figure $7 \mathrm{~b}$. The values of $D_{\mathrm{S}}$ for Nafion agglomerates are

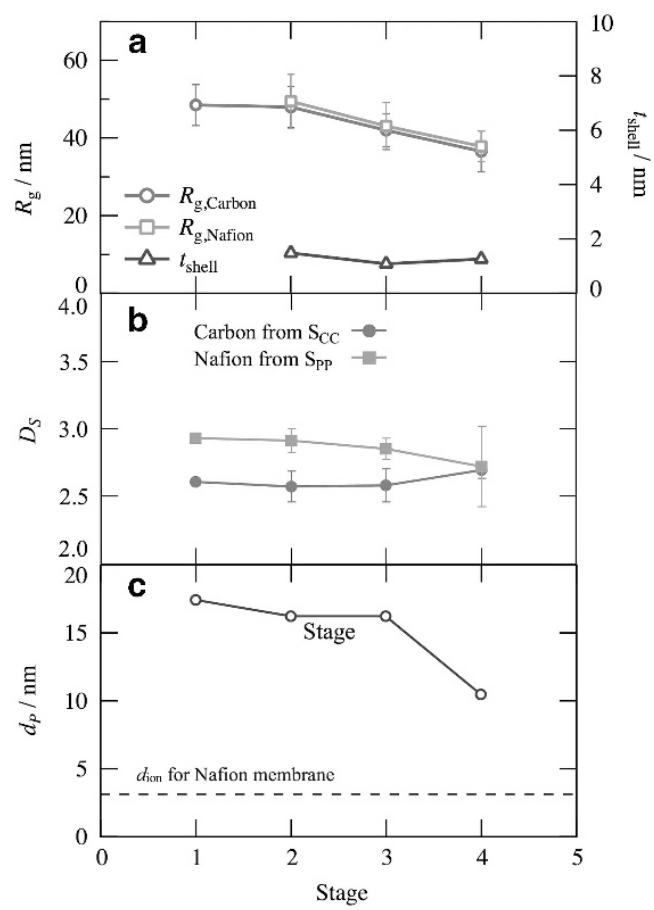

Figure 7 Concentration dependence of (a) the radius of gyration, $R_{\mathrm{g}}$, for carbon $\left(R_{\mathrm{g}, \text { Carbon }}\right)$ and Nafion $\left(R_{\mathrm{g}, \text { Nafion }}\right)$ and the thickness of Nafion shell $\left(t_{\text {shell }}\right)$, (b) the surface fractal dimension, $D_{S}$, obtained from the power law fitting of the SANS partial structure factors for carbon $\left(S_{C C}(q)\right)$ (red circles) and Nafion $\left(S_{\mathrm{pp}}(q)\right)$ (green squares) and (c) the inter-ionic cluster distance, $d_{\mathrm{p}}$. A full color version of this figure is available at Polymer Journal online. higher than that for carbon agglomerates, indicating that the outer surface of the agglomerates is rougher than the inner surface. The two $D_{\mathrm{S}}$ values converged upon drying of the samples, which implies that the Nafion shell is strongly adsorbed to the carbon core to form a similar surface structure. As a matter of fact, both $S_{\mathrm{CC}}(q)$ and $S_{\mathrm{PP}}(q)$ are indistinguishable, except for the large $q$ region where the polyelectrolyte peak appears $\left(0.03<q<0.2 \AA^{-1}\right)$. The variation of the inter-ionic cluster distance $d_{\mathrm{P}}$ during the drying process is shown in Figure $7 \mathrm{c}$. The dashed line shows the value of the inter-ionic channel distance of a dry Nafion membrane $\left(d_{\text {ion }}=3 \mathrm{~nm}\right) .^{5}$ The $d_{\mathrm{P}}$

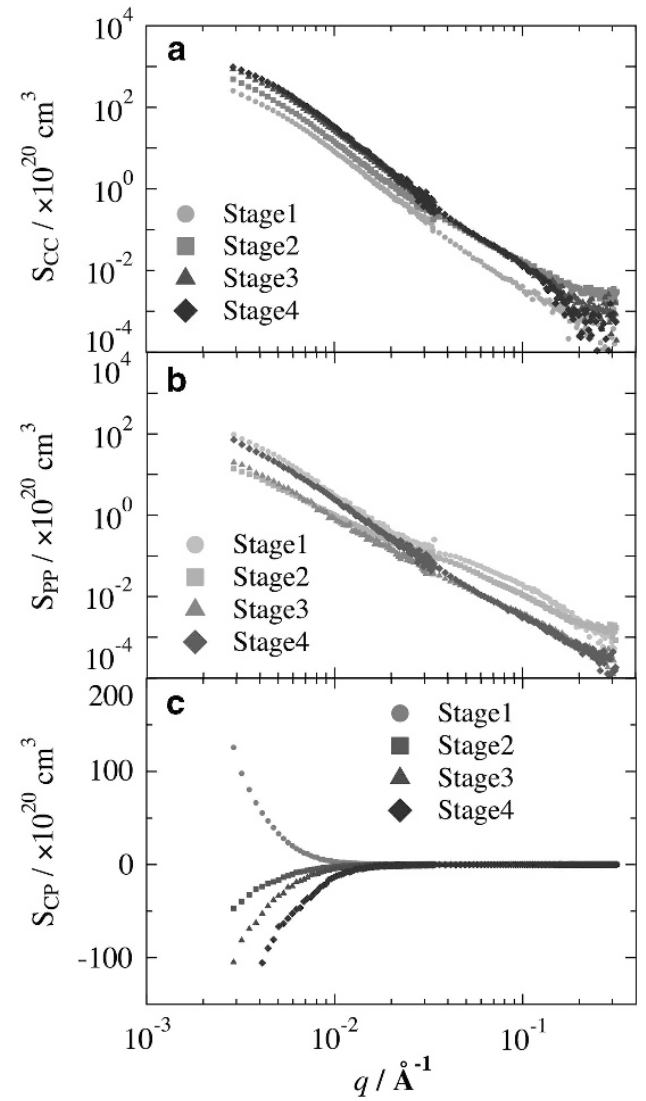

Figure 9 Decomposed partial structure factors, (a) $S_{\mathrm{CC}}(q)$, (b) $S_{\mathrm{pp}}(q)$ and (c) $S_{\mathrm{CP}}(q)$ with varying ink concentrations for the lonomerA systems. A full color version of this figure is available at Polymer Journal online.
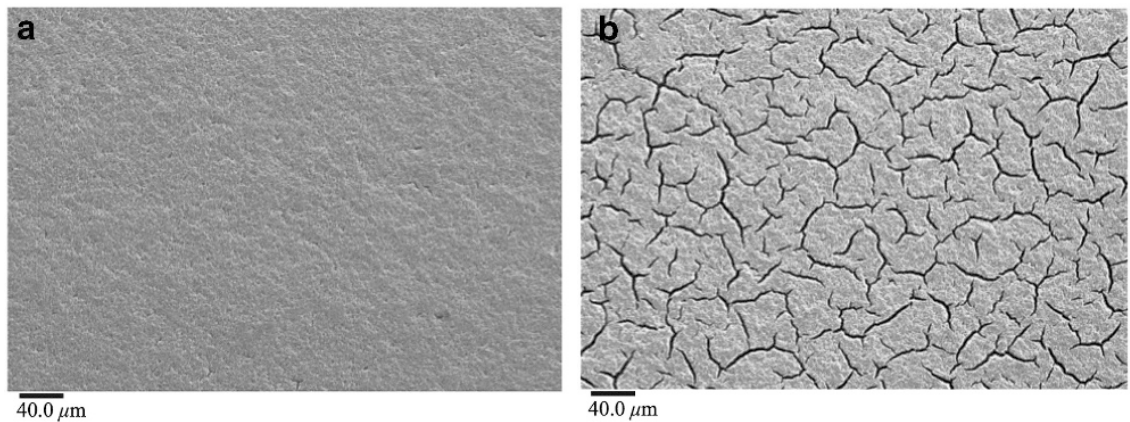

Figure 8 TEM images for electrodes with (a) Nafion and (b) lonomerA. TEM, transmission electron microscopy. 
values decrease and become close to the value of $d_{\text {ion }}(3 \mathrm{~nm})$ upon drying, which is due to an increase in ink concentration.

\section{IonomerA}

Figures $8 \mathrm{a}$ and $\mathrm{b}$ show the transmission electron microscopy micrographs for the electrodes that include (a) Nafion and (b) IonomerA.

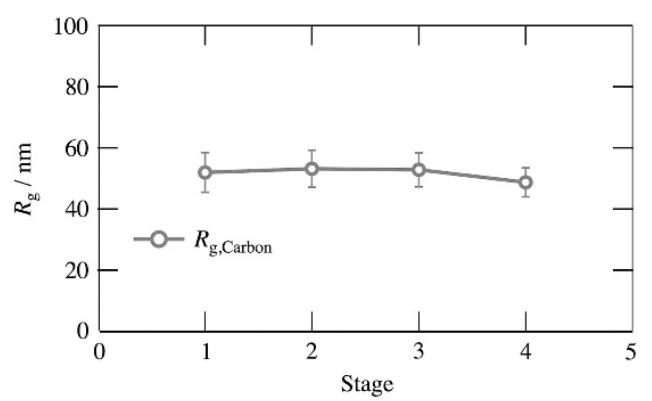

Figure 10 Concentration dependence of the radius of gyration, $R_{\mathrm{g}}$, for carbon $\left(R_{\mathrm{g}, \mathrm{Carbon}}\right)$ for the lonomerA systems. A full color version of this figure is available at Polymer Journal online.
The electrodes with IonomerA exhibited many cracks, which could be explained by a lower molecular interaction between the ionomer and carbon. Figures $9 \mathrm{a}-\mathrm{c}$ show the three partial structure factors, (a) $S_{\mathrm{CC}}(q)$, (b) $S_{\mathrm{PP}}(q)$ and (c) $S_{\mathrm{CP}}(q)$, for the IonomerA systems. Interestingly, $S_{\mathrm{CP}}(q)$ is negative for Stage 2-4, which implies that IonomerA is only found in carbon agglomerates. Note also that $S_{\mathrm{PP}}(q)$ is $\sim 10$ times smaller than $S_{\mathrm{CC}}(q)$ for $q \leqslant 0.03 \AA^{-1}$. However, polyelectrolyte scattering is clearly observed in the $S_{\mathrm{PP}}(q)$ of Stages 1 and 2 for $q \geqslant 0.03 \AA^{-1}$. This result strongly indicates that IonomerA has not penetrated the carbon agglomerates enough in comparison with Nafion. The estimated $R_{\mathrm{g}}$ values from $S_{\mathrm{CC}}(q)$ by the Guinier fitting are shown in Figure 10. The size of the carbon agglomerates was nearly constant during the drying process, which is a clear difference from the case of the Nafion systems.

\section{Structural evolution of the catalyst ink}

On the basis of the experimental results and related discussions, we propose a model for the structural evolution of the catalyst ink during the drying process, which is shown in Figure 11. According to previous studies, ${ }^{16,23}$ the ionomer distribution is not homogeneous. Therefore, the thickness of the ionomer layer also varies widely.

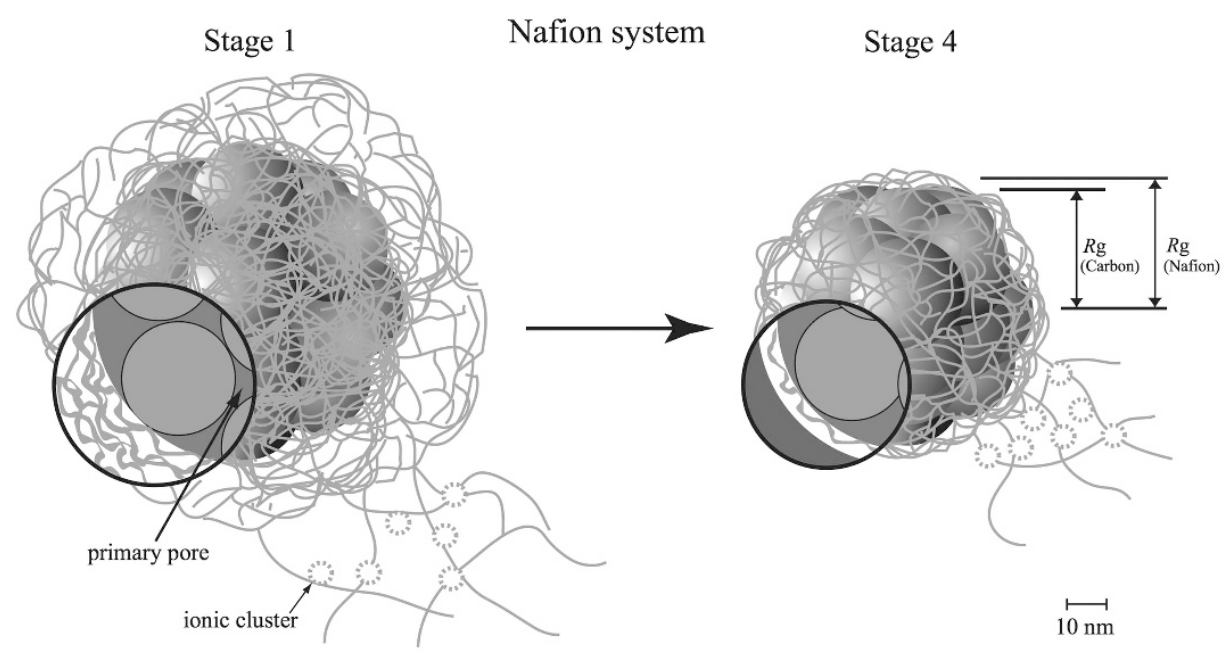

Stage $1 \quad$ IonomerA system

Stage 4

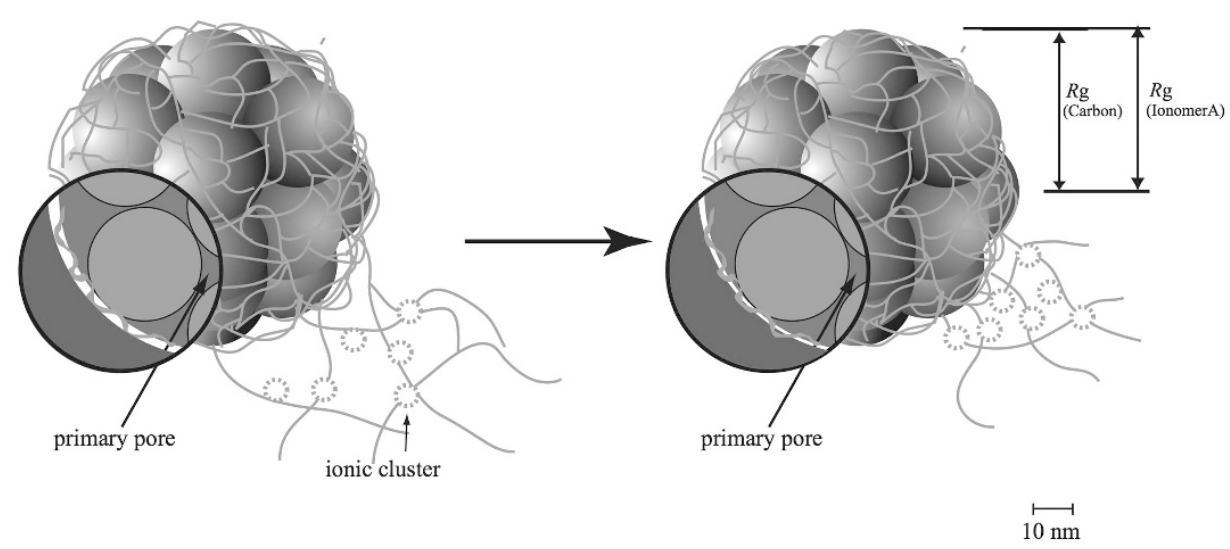

Figure 11 Schematic illustration of the structural change of catalyst ink during the drying process. The carbon agglomerates shrink and the thickness of the Nafion shell decreases upon drying for the Nafion system. A full color version of this figure is available at Polymer Journal online. 
Note that the structure of the catalyst ink observed by SANS is an averaged structure in the irradiated region of the samples. Among the hierarchical structure of the catalyst ink, the agglomerates mainly are observed by SANS. Therefore, we only discussed the structure of the agglomerates in this study. For the catalyst ink, the major components are the carbon particles with a core radius of $\sim 42 \mathrm{~nm}$ and outer shell radius $>50 \mathrm{~nm}$. In this shell region, the ionomer chains are weakly adsorbed to and interpenetrated into the carbon agglomerates. For catalyst ink dried to a concentration ratio of 4 (Stage 4), both the size of the carbon agglomerates and the thickness of the Nafion shell decrease. As mentioned in the Introduction, it has been reported that an agglomerate includes nanopores, called primary pores, between the primary carbon particles. ${ }^{24}$ The shrinking of the agglomerates during the drying process is accompanied by the decrease in primary pore size. In the polymer matrix, ionic channels are observed even at such low concentrations, and the inter-ionic channel distance is evaluated to be $\sim 8 \mathrm{~nm}$, which decreases with increasing concentration. According to Kim et al., ${ }^{5}$ ion channels are present in dried Nafion films. Our results suggest that the ionic clusters persist irrespective of changes in concentration, the inter-ionic cluster distance decreases upon drying and the inter-ionic cluster may eventually percolate and form ion channels by keeping an inter-ionic channel distance of $3 \mathrm{~nm} .{ }^{5}$ In the case of IonomerA, on the other hand, the size of the carbon agglomerates is almost constant during the drying process, indicating the formation of sparse agglomerates upon drying, which the polymer penetrate less. This may be related to the improvement in proton conductivity.

Although many studies have been performed until now, there are no studies, to our knowledge, correlating the structure of the catalyst ink with that of the electrode. In this study, we could elucidate the structural transition of the catalyst ink during the drying process and obtain important information for applications in PEFCs. The resulting SANS profiles provide structural information averaged over the material traversed by the beam and only on the nanoscale. According to ultra-small-angle X-ray scattering or scanning X-ray microscopy studies, ${ }^{16,25}$ there is an inhomogeneous distribution of carbon and ionomers in the catalyst ink or electrode. The evolution of the mesoscale structure including any inhomogeneous distribution or carbon secondary pores during the drying process is also important and is a future work.

\section{CONCLUSION}

CV-SANS experiments were carried out to elucidate the structural change of ionomers (Nafion and IonomerA) and carbon agglomerates during the drying process. The cross term, that is, $S_{\mathrm{CP}}(q)$, indicates that the microscopic structure of the catalyst ink retains the clusters of carbon agglomerates surrounded by Nafion during the drying process. This result is consistent with other previously reported results. ${ }^{4}$ The carbon partial structure factor, $S_{\mathrm{CC}}(q)$, indicates that the size of the carbon agglomerates decreases with solvent evaporation. The Nafion partial structure factor, $S_{\mathrm{PP}}(q)$, indicates that Nafion effectively interpenetrated the carbon agglomerates, and the thickness of the Nafion shell decreases during the drying process. The shrinking of the carbon agglomerates and the Nafion shell is attributed to the interaction between carbon and the Nafion shell. For the IonomerA systems, $S_{\mathrm{CP}}(q)$ was negative for Stage 2-4, which implies that IonomerA does not penetrate the carbon agglomerates. Electrodes with IonomerA exhibited many cracks, which could be explained by the low interaction between IonomerA and carbon. Furthermore, the size of the carbon agglomerates was almost constant during the drying process, resulting in the formation of sparse agglomerates. This was also due to the low interaction between IonomerA and carbon. These findings are important for the design and precise control of catalyst inks towards fuel cell applications although the concentrations are limited to only 3 to $11 \mathrm{wt} \%$. It was confirmed that ionic clusters are present even at 1.5 wt $\%$ and that the inter-ionic cluster distance decreases upon drying. The ionic clusters may percolate as the concentration increases and engage in ion conduction. The confirmation of this hypothesis is a topic for future studies.

\section{ACKNOWLEDGEMENTS}

The SANS experiment was performed by using a $40 \mathrm{~m}$ SANS instrument at the High-flux Advanced Neutron Application Reactor (HANARO), Korea Atomic Energy Research Institute (KAERI), Daejeon, South Korea, which was transferred from SANS-U at JRR-3 with the approval of the Institute for Solid State Physics, The University of Tokyo (proposal no. 13596), Japan Atomic Energy Agency, Tokai, Japan. We are grateful to T. W. Kim and Y. S. Han for helpful assistance in the SANS experiments.

1 Litster, S. \& McLean, G. PEM fuel cell electrodes. J. Power Sources 130 61-76 (2004).

2 Uchida, M., Aoyama, Y., Eda, N. \& Ohta, A. New preparation method for polymerelectrolyte fuel cells. J. Electrochem. Soc. 142, 463-468 (1995).

3 Uchida, M., Fukuoka, Y., Yasushi Sugawara, Y., Ohara, H. \& Ohta, A. Improved preparation process of very- low-Plati flu rn-loading electrodes for polymer electrolyte fuel cells. J. Electrochem. Soc. 145, 3708-3713 (1998).

4 Holdcroft, H. Fuel cell catalyst layers: a polymer science perspective. Chem. Mater. 26 381-393 (2014).

5 Kim, M.-H., Glinka, C. J., Grot, S. A. \& Grot, W. G. SANS study of the effects of water vapor sorption on the nanoscale structure of perfluorinated sulfonic acid (NAFION) membranes. Macromolecules 39, 4775-4787 (2006).

6 Iwase, H., Koizumi, S., likura, H., Matsubayashi, M., Yamaguchi, D., Maekawa, Y. \& Hashimoto, T. A combined method of small-angle neutron scattering and neutron radiography to visualize water in an operating fuel cell over a wide length scale from nano to millimeter. Nucl. Inst. Method Phys. Res. A 605, 95-98 (2009).

7 Sekhon, S. S., Park, J.-S., Baek, J.-S., Yim, S.-D., Yang, T.-H. \& Kim, C.-S. Small-angle $X$-ray scattering study of water free fuel cell membranes containing ionic liquids. Chem. Mater. 22, 803-812 (2010).

$8 \mathrm{He}, \mathrm{Q}$., Suraweera, N. S., Joy, D. C. \& Keffer, D. J. Structure of the ionomer film in catalyst layers of proton exchange membrane fuel cells. J. Phys. Chem. C 117 25305-25316 (2013).

9 Aldebert, P., Dreyfus, B. \& Pineri, M. Small-angle neutron scattering of perfluorosulfonated ionomers in solution. Macromolecules 19, 265102653 (1986).

10 Gebel, G. Structural evolution of water swollen perfluorosulfonated ionomers from dry membrane to solution. Polymer 41, 5829-5838 (2000).

11 Gebel, G. \& Diat, O. Neutron and X-ray scattering: suitable tools for studying ionomer membranes. Fuel Cells 5, 261-276 (2005).

12 Gebel, G., Lyonnard, S., Mendil-Jakani, H. \& Morin, A. The kinetics of water sorption in Nafion membranes: a small-angle neutron scattering study. J. Phys. Condens. Matter 23 (2011).

13 Malek, K., Eikerling, M., Wang, Q. P., Navessin, T. C. \& Liu, Z. S. Self-organization in catalyst layers of polymer electrolyte fuel cells. J. Phys. Chem. 111, 13627-13634 (2007).

14 More, K., Borup, R. \& Reeves, K. Identifying contributing degradation phenomena in PEM fuel cell membrane electride assemblies via electron microscopy. ECS Trans. 3, 717-733 (2006).

15 Paul, D. K., Karan, K., Docoslis, A., Giorgi, J. B. \& Pearce, J. Characteristics of selfassembled ultrathin Nafion films. Macromolecules 46, 3461-3475 (2013).

16 Berejnov, V., Susac, D., Stumper, J. \& Hitchcock, A. P. 3D chemical mapping of PEM fuel cell cathodes by scanning transmission soft X-ray spectrotomography. ECS Trans. 50, 361-368 (2013).

17 Shibayama, M., Matsunaga, T., Kusano, T., Amemiya, K., Kobayashi, N. \& Yoshida, T. SANS studies on catalyst ink of fuel cell. J. Appl. Polym. Sci. 131, 39842 (2014).

18 Endo, H., Schwahn, D. \& Cölfen, J. On the role of block copolymer additives for calcium carbonate crystallization: Small angle neutron scattering investigation by applying contrast variation. J. Chem. Phys. 120, 9410-9423 (2004).

19 Miyazaki, S., Endo, H., Karino, T., Haraguchi, K. \& Shibayama, M. Gelation mechanism of Poly(N-isopropylacrylamide)-clay nanocomposite gels. Macromolecules 40 4287-4295 (2007). 
20 Endo, H., Mihailescu, M., Monkenbusch, M., Allgaier, J., Gompper, G., Richter, D., Jakobs, B., Sottmann, T., Strey, R. \& Grillo, I. Effect of amphiphilic block copolymers on the structure and phase behavior of oil-water-surfactant mixtures. J. Chem. Phys. 115, 580-600 (2001).

21 Borue, V. \& Erukhimovich, I. A statistical theory of weakly charged polyelectrolytes: fluctuations, equation of state, and microphase separation. Macromolecules 21, 3240-3249 (1988).

22 Higgins, J. S. \& Benoit, H. C. Polymers and Neutron Scattering, (Clarendon Press, Oxford, UK, 1994)
23 Lee, M., Uchida, M., Yano, H., Tryk, D. A., Uchida, H. \& Watanabe, M. New evaluation method for the effectiveness of platinum/carbon electrocatalysts under operating conditions. Electrochim. Acta 55, 8504-8512 (2010).

24 Soboleva, T., Zhao, X. S., Mallek, K., Xie, Z., Navessin, T. \& Holdcroft, S. On the micro-, meso-, and macroporous structures of polymer electrolyte membrane fuel cell catalyst layers. ACS Appl. Mater. Interfaces 2, 375-384 (2010).

25 Xu, F., Zhang, H. Y., Ilavsky, J., Stanciu, L., Ho, D., Justice, M. J., Petrache, H. I. \& Xie, $\mathrm{J}$. Investigation of a catalyst ink dispersion using both ultra-small-angle $\mathrm{X}$-ray scattering and cryogenic TEM. Langmuir 26, 19199-19208 (2010). 